\title{
Researchers' views on return of incidental genomic research results: qualitative and quantitative findings
}

\author{
Robert Klitzman, MD1, Paul S. Appelbaum, MD1, Abby Fyer, MD¹, Josue Martinez, BA², \\ Brigitte Buquez, BA 1 , Julia Wynn, $\mathrm{MS}^{2}$, Cameron R. Waldman, BA ${ }^{3}$, Jo Phelan, PhD ${ }^{4}$, Erik Parens, PhD ${ }^{3}$ \\ and Wendy K. Chung, MD, PhD 2,5
}

Purpose: Comprehensive genomic analysis including exome and genome sequencing is increasingly being utilized in research studies, leading to the generation of incidental genetic findings. It is unclear how researchers plan to deal with incidental genetic findings.

Methods: We conducted a survey of the practices and attitudes of 234 members of the US genetic research community and performed qualitative semistructured interviews with 28 genomic researchers to understand their views and experiences with incidental genetic research findings.

Results: We found that $12 \%$ of the researchers had returned incidental genetic findings, and an additional $28 \%$ planned to do so. A large majority of researchers (95\%) believe that incidental findings for highly penetrant disorders with immediate medical implications should be offered to research participants. However, there was no consensus on returning incidental results for other conditions varying in penetrance and medical actionability. Researchers raised concerns that the return of incidental findings would impose significant burdens on research and could potentially have deleterious effects on research participants if not performed well. Researchers identified assistance needed to enable effective, accurate return of incidental findings.

Conclusion: The majority of the researchers believe that research participants should have the option to receive at least some incidenta genetic research results.

Genet Med advance online publication 27 June 2013

Key Words: genomic results; incidental findings; return of results; secondary findings; whole-exome sequencing

predictive of future illness may also create anxiety in participants and family members, and require further evaluation.

Even in the face of these concerns, many researchers and institutional review boards feel obliged to offer to return results that could avert death or significant morbidity. ${ }^{3,4}$ Consensus is lacking, however, on which IFs should be returned ${ }^{5}$ and on how clinical utility should be defined. ${ }^{6-9}$ Unique considerations arise regarding return of IFs to special research populations, including pregnant women following prenatal diagnosis, minors, deceased research participants, and participants lacking capacity.

Return of IFs could significantly affect the research enterprise and participants' well-being. Although researchers' views alone will not determine policies on return of IFs, they are likely to have important perspectives on the feasibility, costs, and consequences of various approaches. Moreover, policies at significant variance with the moral intuitions of most researchers may not be implemented effectively. To ensure that policy in this area takes into account the views of the research community, we conducted an Internet-based survey and a smaller number of in-depth interviews to elicit genetic researchers' opinions about return of IFs. 


\section{MATERIALS AND METHODS}

\section{Participants}

We identified genetic researchers by (i) searching the National Institutes of Health online RePORTER database for principal and coprincipal investigators of currently funded grants using combinations of key words (e.g., human genetics, human genomics, genetic epidemiology, exome sequencing, whole-genome sequencing, and genome-wide association) and (ii) applying similar criteria to the abstracts from the 2011 American Society of Human Genetics meeting. Only investigators whose research focus was human disease-gene identification were included. E-mail addresses were identified using online resources. Individuals outside the United States and for whom no e-mail address was found were excluded. Ninety-three researchers were identified using the criteria described above and invited to participate in a telephone interview. Twenty-five researchers responded affirmatively, were interviewed, and were excluded from the online survey (response rate $=56 \%$ ). Of the remaining 787 researchers invited to participate in the survey, 30 e-mail addresses were incorrect, and 23 researchers indicated that they were not conducting relevant research. A total of 254 of the remaining 734 researchers responded to the survey (34.7\%). Thirteen responses were excluded because respondents answered $<50 \%$ of the questions. Three survey respondents indicated willingness to participate in the interviews and were added to those interviewed.

\section{Instruments}

The survey included multiple-choice questions and opportunities to enter comments. Content was based on a literature review and the initial interviews with researchers, and was designed to elicit attitudes and experiences. It was reviewed by six researchers, two genetic counselors, and two research coordinators, with subsequent revisions, and was piloted with 10 researchers. It took $20 \mathrm{~min}$ to complete. The interviews covered similar questions as the survey, in a semistructured interview format, lasting about an hour.

\section{Procedures}

Researchers eligible for the survey were contacted by e-mail to solicit their participation. They were invited to click on a link, where they viewed an informed consent disclosure. E-mail reminders were sent twice to nonrespondents. Respondents were offered a $\$ 25$ gift certificate for participation and could skip any questions they did not wish to answer. The survey was conducted between August and October 2012.

The interviews were conducted by telephone during the same period. Researchers who indicated a willingness to be interviewed were e-mailed a consent form; verbal consent was obtained before beginning the interview. The study was approved by the institutional review boards of Columbia University Medical Center and the New York State Psychiatric Institute.

\begin{tabular}{|c|c|}
\hline Demographic characteristic & Value \\
\hline Male & $64.3 \%$ \\
\hline Age & $43.2 \pm 11.2$ \\
\hline \multicolumn{2}{|l|}{ Race/ethnicity } \\
\hline Asian & $14.5 \%$ \\
\hline Black or African American & $0.4 \%$ \\
\hline Hispanic & $5.0 \%$ \\
\hline Non-Hispanic white & $73.0 \%$ \\
\hline More than one race & $1.7 \%$ \\
\hline Unknown or not reported & $5.4 \%$ \\
\hline \multicolumn{2}{|l|}{ Education } \\
\hline MD & $19.1 \%$ \\
\hline $\mathrm{PhD}$ & $51.9 \%$ \\
\hline $\mathrm{MD} / \mathrm{PhD}$ & $13.3 \%$ \\
\hline MS & $7.9 \%$ \\
\hline Other & $7.9 \%$ \\
\hline
\end{tabular}

\section{Data analysis}

Survey responses are provided in aggregate form and are characterized with descriptive statistics. Interviews were coded and analyzed using grounded theory, ${ }^{10}$ seeking to obtain a "thick description." ${ }^{11}$ Blocks of text were assigned "core" codes or categories (e.g., reasons to return or not return IFs), with reconciliation of independently developed coding schemes and preparation of a coding manual. Principal subcategories were identified (e.g., reasons not to return IFs, related to costs of Clinical Laboratory Improvement Amendments confirmation), and codes and subcodes were developed that were used in analysis of the interviews by two coders.

\section{Description of samples}

\section{RESULTS}

Survey respondents (Table 1 ) had a mean age of 43.2 years (SD 11.2 years) and were predominantly male and non-Hispanic Caucasian. Respondents included PhDs (51.9\%), MDs (19.1\%), and $\mathrm{MD} / \mathrm{PhDs}(13.3 \%)$, and they performed many roles in research, including obtaining informed consent, collecting phenotypic data and biospecimens, analyzing genomic data, and providing clinical care to participants (Table 2).

Research areas were diverse, with almost half involved in neurological or psychiatric disorders, as well as cancer; birth defects; obesity; diabetes; asthma, pharmacogenetics; and cardiac, autoimmune, infectious, hearing, and ophthalmologic disorders. Almost all respondents study adults; more than half study children; and nearly $30 \%$ study patients with conditions that may be fatal before the studies end (Table 2). Respondents reported using a variety of genetic methods in their research, including copy number-variant analysis (68\%), WES (73.9\%), WGS (54.8\%), and both WES and WGS (46.5\%). Of researchers 
not currently performing WES/WGS, virtually all (96\%) anticipate using these techniques in the future. Of the 204 researchers who had used WES/WGS, $49.6 \%$ had sequenced $>100$ and $13.3 \%$ had sequenced $>1,000$ research participants.

Table 2 Roles of researchers and their research studies for respondents who completed the online survey

\begin{tabular}{|c|c|c|}
\hline Researcher roles and characteristics & Number & Percentage \\
\hline \multicolumn{3}{|l|}{ Role(s) of the researcher } \\
\hline Obtaining informed consent & 116 & 48.1 \\
\hline $\begin{array}{l}\text { Collection of clinical/phenotypic data and } \\
\text { biospecimens }\end{array}$ & 131 & 54.4 \\
\hline Generating genomic data & 164 & 68.0 \\
\hline Analysis of genomic data & 218 & 90.5 \\
\hline Receiving deidentified samples/data & 194 & 80.5 \\
\hline Providing clinical care & 14 & 5.8 \\
\hline \multicolumn{3}{|l|}{ Years of experience in human genetic research } \\
\hline$<1$ year & 4 & 2.3 \\
\hline $1-5$ years & 54 & 30.5 \\
\hline $6-10$ years & 42 & 23.7 \\
\hline $11-20$ years & 48 & 27.1 \\
\hline$>20$ years & 29 & 16.4 \\
\hline \multicolumn{3}{|l|}{ Populations studied } \\
\hline Adults & 228 & 94.6 \\
\hline Children & 137 & 56.8 \\
\hline Fetuses & 20 & 8.3 \\
\hline Adults lacking decision-making capacity & 41 & 17.0 \\
\hline Terminally ill & 72 & 29.9 \\
\hline \multicolumn{3}{|l|}{ Number of participants enrolled } \\
\hline$\leq 100$ & 29 & 16.3 \\
\hline $101-500$ & 29 & 16.3 \\
\hline $501-1,000$ & 20 & 11.2 \\
\hline $1,001-5,000$ & 66 & 37.1 \\
\hline $5,001-10,000$ & 15 & 8.4 \\
\hline$>10,000$ & 19 & 10.7 \\
\hline \multicolumn{3}{|l|}{ Genetic methods used } \\
\hline Candidate gene resequencing & 176 & 73.0 \\
\hline CNV analysis & 164 & 68.0 \\
\hline GWAS & 164 & 68.0 \\
\hline WES & 178 & 73.9 \\
\hline WGS & 132 & 54.8 \\
\hline WES and WGS & 112 & 46.5 \\
\hline Plans to do WES/WGS & 35 & 14.5 \\
\hline \multicolumn{3}{|l|}{ Participants studied using WES or WGS } \\
\hline$<10$ & 25 & 12.3 \\
\hline $11-50$ & 41 & 20.1 \\
\hline $51-100$ & 32 & 15.7 \\
\hline $101-500$ & 54 & 26.5 \\
\hline $501-1,000$ & 20 & 9.8 \\
\hline$>1,000$ & 32 & 13.3 \\
\hline
\end{tabular}

CNV, copy-number variant; GWAS, genome-wide association study; WES, wholeexome sequencing; WGS, whole-genome sequencing.
Thirty-seven percent of respondents reported that their consents allow for return of genetic research results that are the primary focus of the study, and $28 \%$ had in fact returned such results. Twenty-six percent responded that their consents allow for return of IFs, and $12 \%$ had returned IFs. In the future, an additional $28 \%$ plan to disclose IFs, and 30\% plan to re-obtain consent from participants in WES/WGS.

The 28 interviewees included 11 women and 17 men, 8 MDs, $14 \mathrm{PhDs}, 4 \mathrm{MD} / \mathrm{PhDs}$, and 2 with MS degrees. Nineteen had performed WES and/or WGS, and three anticipated doing so. Fifteen had generated IFs, and 11 had returned IFs.

Below we integrate the survey and interview results. For survey questions that asked for agreement with statements on a $1-5$ scale, we indicate mean scores and SDs. Where quotations are given, subject identification numbers indicate interview quotations ("R-I") and survey free-text responses ("R-S").

\section{Attitudes toward return of IFs}

Forty-nine percent survey respondents felt that participants should be given the option of deciding whether to have IFs returned, and $38.3 \%$ thought that participants probably should have this option. Only $12.9 \%$ thought that participants should not have this option. And $79.8 \%$ felt that participants should be given the option of deciding what types of IFs are returned.

\section{Reasons to return IFs}

When asked, "On a scale of 1-5 how important would each of the following reasons be for returning incidental results?" respondents said the most important reason was "because the information could be life-saving and that there would be a moral obligation to return such life-saving information to participants" $(4.3 \pm 1.0)$ (Figure 1). Many researchers believed that to withhold such medically actionable information is morally uncomfortable. Researchers, especially if also clinicians (even if not directly providing care to participants), may feel a degree of clinical responsibility to their research participants. One project manager who had worked with clinician and nonclinician researchers noted that clinician-researchers showed a stronger willingness to return results of clinical significance. "They would say, "Look, these are my patients. I'm going to tell them"'” (R-I 5).

The second most important reason researchers gave for returning results was that participants had a right to IFs $(3.9 \pm 1.2)$ (Figure 1) (e.g., "A person owns their own genome. If they want to know what their genome is ... they have a right to know, period. The implications of the knowledge don't matter" (R-I 22)). In answering questions, researchers often used themselves as a standard. A researcher who supported returning all variants to participants said, "If it were me, I would want to know everything" (R-I 25).

Concerns about potential liability played less prominent roles in researchers" views $(3.1 \pm 1.3)$. One researcher had "concern about legal implications of doing/not doing something. Seems [it] could open up liability even if we did nothing because it could be argued we should have done something. So there is 


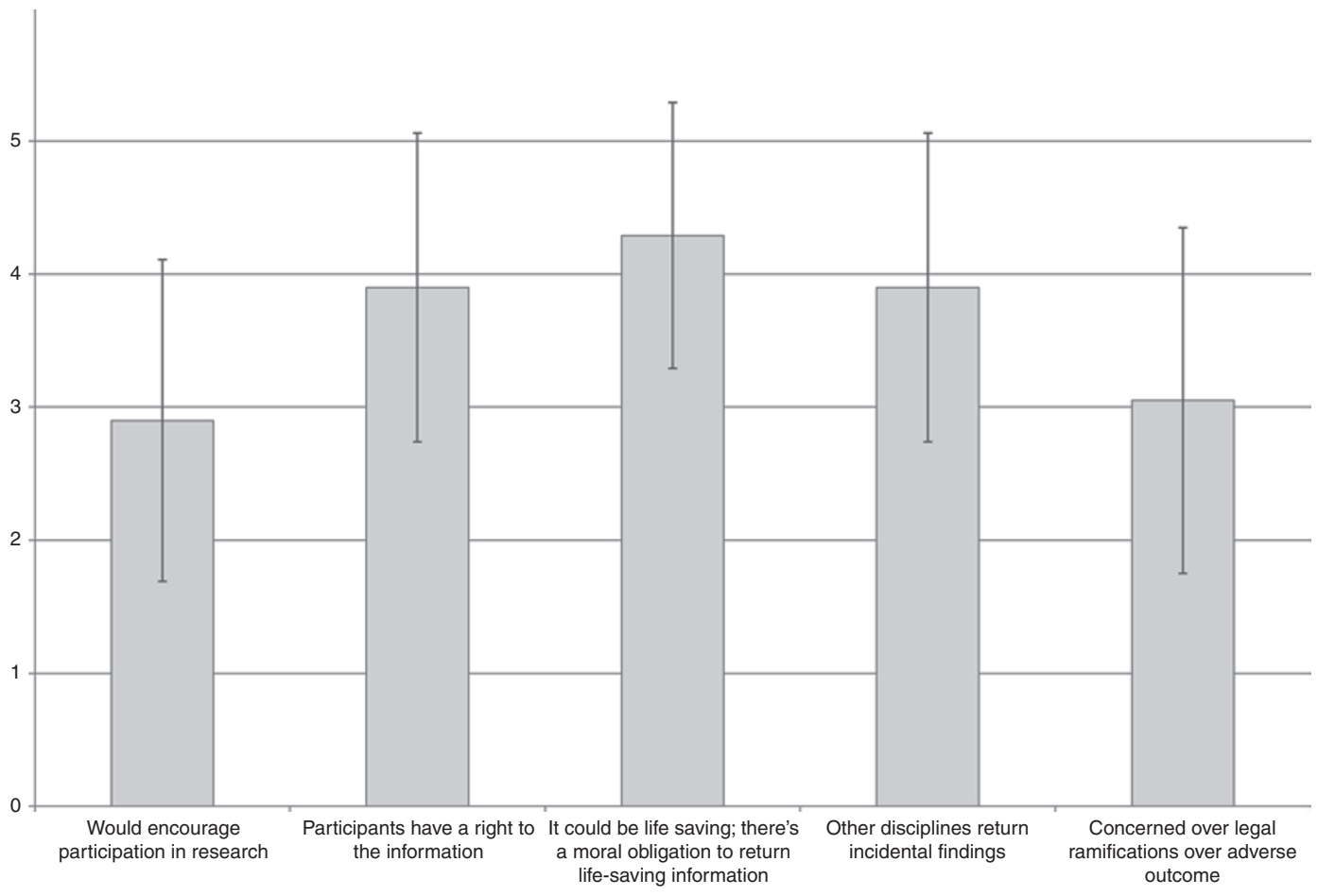

Figure 1 Mean importance of each item to consider for returning incidental genetic findings in research. Scale from 1-5, with 5 representing most important. Vertical lines indicate one SD above and below the mean.

safety in not being allowed to do anything" (R-S 118). Other reasons offered for returning findings were that other disciplines (e.g., radiology) return IFs that have medical implications $(2.9 \pm 1.2)$ and that it would encourage research participation $(2.9 \pm 1.2)$.

I think that returning of incidental results is often appreciated by research subjects as a way of demonstrating that the researchers care about the benefit to them and not just the benefit to the research (R-S 1).

\section{Reasons not to return IFs}

Researchers also raised varied reasons not to return IFs, most importantly that most results still have largely uncertain clinical implications $(4.1 \pm 1.2)$ (Figure 2). Several researchers felt returning uncertain findings could be detrimental to participants.

If we don't know what something means, we're doing more of a disservice than a service by telling patients .... Our credo is "do no harm." Sometimes telling people something that you don't understand does harm (R-I 17).

Others expressed concern with the quality of current sequencing technology and data. "I would never return, even think of returning something that's coming out of nextgen[eration] sequencing right now" (R-I 9). Researchers were similarly concerned about not having staff capable of explaining results to participants $(3.2 \pm 1.4)$. Several believed results were best returned and explained by professionals with clinical training that enabled them to "connect" with participants: "As researchers, we are not equipped to offer [to] return findings to patients" (R-S 182).

Lack of institutional review board permission to return IFs was another important consideration (3.2 \pm 1.4$)$. Institutional review board-approved consent forms vary in whether and how they discuss IFs, e.g., informing participants that uncertain results will not be returned but failing to anticipate that these uncertain findings might later be found to have medical significance.

Researchers also expressed concerns that their labs were not Clinical Laboratory Improvement Amendments-approved, and that confirmation in a certified laboratory can be expensive, with the source of funding unclear $(3.2 \pm 1.4)$. "We just don't have the money to go back and retest this, to reanalyze these sequences, to see if people have these variants .... It comes down to cost" (R-I 5). Lack of expertise to know what results should be returned was also important, although less so $(2.9 \pm 1.4)$ : "Subjects should have the option of obtaining the results, but it can't be expected of a researcher (e.g., studying neurological disorders) to know the relevance of variants they found for cancer, etc." (R-S 106). Researchers also worried that the return of incidental results would distract the laboratory from the primary task of performing research $(2.8 \pm 1.4)$. Overall, if return of at least some incidental results were required, $93.7 \%$ of respondents felt that this would be a burden on researchers, and $44 \%$ thought it would be a very heavy or at least a significant burden. As one said, "I'd have to 


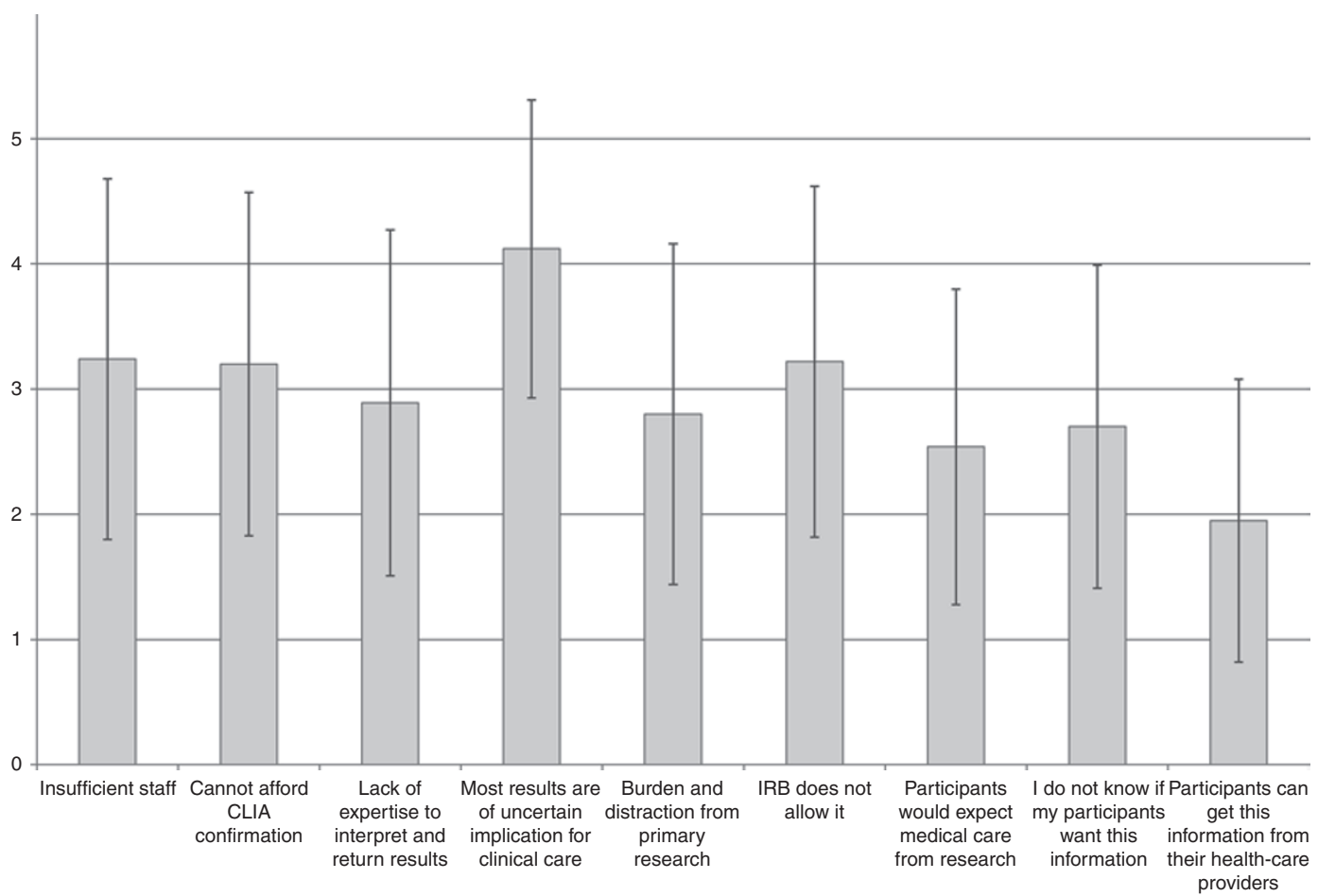

Figure 2 Mean importance of each item to consider for not returning incidental genetic findings in research. Scale from 1-5, with 5 representing most important. Vertical lines indicate one SD above and below the mean. IRB, institutional review board.

do $80 \%$ less research. If you told me I could do only $5 \%$ less research, I'd be really interested" (R-I 18). Another added, "The number of potential IFs is essentially infinite. The amount of overhead for identifying and reporting incidental clinical findings would destroy the research enterprise in genetics" (R-I 28). These investigators worried that decrements in research would impede the development of long-term population benefits. "If we cut back deeply on the research, we won't get to that point in the future where everybody's genome will be sequenced and become part of standard clinical care, rather than a research project" (R-I 18).

Although this was not explicitly probed in the survey, some researchers expressed concern that subjects might not understand the results:

Subjects will inevitably be incapable of distinguishing the various shades of gray relating to potential false-positive and false-negative findings. By way of analogy, in my experience with brain imaging research, the routine practice of clinical reading of research scans has a tendency to do more harm (in terms of cost, as well as time devoted to overcoming the natural therapeutic misconception that arises) than good. There are almost never any clinically actionable results, but there are not infrequent incidental findings of minimal clinical significance that nevertheless require worry-making follow-up (R-S 077).

Several expressed vociferous opposition to returning IFs. "I feel very strongly that the field is heading over the cliff on this issue ... incidental findings should not be returned to subjects-full stop" (R-S 077).

\section{Unsure/mixed views}

Given these competing moral and logistical arguments, many researchers were uncertain how to proceed, feeling that they were engaged in a complex "balancing act." Even within the same team, researchers disagreed. Many felt that subjects ideally should be given the option of deciding whether they want IFs returned but that countervailing factors would make it nearly impossible to provide this information adequately. Many researchers tried to balance participants' rights to information versus responsibilities not to harm subjects. "Does a patient have a right to all their information? Of course. But we also have an obligation to not treat it as simple and straightforward, and to try to get across that we can be misled to their detriment if we're not careful" (R-I 26).

Researchers may weigh participants' moral claims against limited resources that could be better used to advance science more broadly.

Ethical positions aside, the true barrier here is that few laboratories will have the resources to provide incidental findings and proper counseling to participants unless the number/type are limited to just those that are clearly actionable right now. And even then, it is going to present a substantial amount of work (R-S 187).

Ultimately, researchers may recognize limits to how much they can pursue certain moral goals. 


\section{What to return}

Although there was wide agreement about some types of IFs to be offered to participants, disagreements arose concerning specific categories. A few researchers said that providing subjects with everything (a "data dump") or nothing would be easiest, not requiring any nuanced decisions. "The gray zone is just too difficult to navigate" (R-I 4). A more common approach was to identify categories or "bins" of findings that justified return. However, some researchers felt that only a small number of bins (three to four) would be manageable (R-I 11), whereas others thought that they should offer more options (e.g., up to 30). Difficult trade-offs appeared because offering too many categories might burden participants. "Thirty choices would be a tough informed consent process to go through. To ask people to make 30 separate decisions would wear them out" (R-I 20).

When asked "If you were going to return IFs (assuming that results were confirmed in a CLIA [Clinical Laboratory Improvement Amendments]-certified laboratory), which of the following categories of data would you consider offering subjects?" researchers largely responded that conditions with high penetrance for which clinical interventions were available should be reported to research participants of all ages (Table 3). But questions arose concerning the definition of "high penetrance." "Is it a $65 \%$ chance of it impacting the person's health, or are we just going to tell him when they have a 98\% [risk]?" (R-I 13). Interviewees also had questions about how to define clinical "actionability," i.e., how good the data and treatment need to be. Some anticipated establishing very high, "absolute" thresholds of actionability, e.g., the disease is $100 \%$ curable. Other researchers thought that the existence of a treatment, even if not fully effective, was sufficient. Lack of health insurance among some participants complicated the picture. "For colorectal and breast cancer the "treatment" is increased screening. If these folks don't have insurance, that becomes a problem" (R-I 5). Some researchers considered interventions such as those to reduce breast cancer risk problematic: "The prophylactic treatments are not so wonderful that I feel compelled to call. But again this is very subjective" (R-I 4).

Many researchers felt, although less strongly, that participants should be offered results for highly penetrant conditions without available interventions (e.g., Huntington disease), and a substantial minority would consider returning IFs for modestly penetrant conditions without interventions (e.g., Alzheimer's disease) (Table 3). Here, the possibility of returning results with personal, but not medical, actionability emerged. Several researchers felt that subjects might want to know of their genetic susceptibilities even if not actionable (e.g., ApoE genotype). Other researchers posed questions about personal actionability and the value of the information in helping patients plan their lives: "You can argue that you could benefit-you'd live your life differently, write your power of attorney differently" (R-I 6).

Regarding genetic results with solely reproductive implications, a majority of researchers said that they would consider offering IFs on carrier status for autosomal recessive conditions to participants of and past reproductive age (Table 3). Some felt that most parents of children who came for testing would want such information. Other researchers might not return such results unless both parents carried a mutation. Although some researchers assumed that participants beyond reproductive age would not want the findings, others pointed out that participants may have relatives of reproductive age who would benefit.

Respondents were split concerning pharmacogenetic variants, and only a minority would return information about variants without clinical consequence (e.g., ancestry). Few would consider offering a list of all variants.

Researchers felt that the participant's age was a factor to consider: $62.1 \%$ and $47.2 \%$ said that there were circumstances in which IFs should be returned for children and for fetuses, respectively; and $51.9 \%$ believed that at some age children should provide assent, suggesting a mean age of $13.66( \pm$ $2.67)$ years. A large majority of respondents $(67.7 \%)$ said that for pediatric participants, data on clinically actionable adultonset conditions with high penetrance (e.g., BRCA1/2) should

Table 3 Percentage of researchers who would be willing to return different types of incidental genetic findings to participants

Data researchers would offer to return to participants of different ages

\begin{tabular}{|c|c|c|c|}
\hline Type of data & Adults, \% & Children, \% & Fetuses, \% \\
\hline High penetrance, with clinical intervention & 95.0 & - & 78.7 \\
\hline Clinically actionable before adulthood & - & 91.5 & - \\
\hline High penetrance, without clinical intervention & 60.2 & 48.5 & 63.3 \\
\hline Modest penetrance, with clinical intervention & 79.3 & - & 60.7 \\
\hline Reproductive implications for prospective parents & 79.3 & 58.3 & 52.7 \\
\hline Reproductive implications for the children of participants & 65.6 & - & - \\
\hline Data on pharmacogenetic variants & 54.4 & 51.9 & 40.7 \\
\hline Potentially relevant, no clinical implications (ancestry) & 21.2 & 14.8 & - \\
\hline List of all variants from entire genome/exome & 15.8 & 14.0 & 8.2 \\
\hline
\end{tabular}


be offered. Some researchers felt that such information might immediately benefit the family and therefore justify providing the information. However, one researcher noted, "I do not think it is worth returning results to children just because the parent may have benefit .... Instead if the parent was interested in learning about themselves, they should participate on their own terms" (R-S 239).

\section{Who should return IFs}

A large majority of the researchers (70.2\%) responded that participants should be offered referrals to genetic counselors or other medical professionals to receive IFs, and $22.7 \%$ suggested that genetic counseling should be provided by the research study; $75.6 \%$ believed that participants should have the option of deciding whether IFs were placed in medical records. Fortyfour percent endorsed the idea that researchers who receive deidentified data should return IFs to the investigators who had primary contact with the participants, allowing that team to decide whether to return such findings.

\section{Who should decide whether IFs are returned}

A large majority of researchers (81.5\%) felt that participants should have the option of deciding whether they want IFs returned. When asked if they would ever override participants' decisions, only $11.8 \%$ would, whereas $32.8 \%$ were unsure. Those who said that they would override a participant's decision often highlighted highly penetrant, medically actionable conditions: "Incidental findings with clinical utility, e.g., $B R C A 1$ and $B R C A 2$, should be disclosed, period, and the participant should be told to expect a call if those show up. They should not be deciding [for themselves and relatives] to ignore it" (R-S 151). Many researchers, however, felt participants' choices should be respected, even if it meant not returning clinically important results: "If you present it to them and they say no, then the answer is no" (R-I 9).

\section{Perceptions of research participants' views}

Researchers sensed that many participants who elected to receive IFs might not understand the implications.

Most people don't think hard about the implications. The casual comment "I want everything" is usually not so reflective of what people want once they've really thought and been educated about it (R-I 26).

When informed, participants may change their minds. "When you talk for 15 seconds about whether they really want information [about conditions that] they can't prevent or treat, many people change their mind" (R-I 10).

Respondents also expressed concern that, if no results are returned, participants may assume that they are being given a "clean bill of health." "In most cases, people do not get a result .... So people think that they're fine" (R-I 28). Some participants may expect results, even if told that results are only rarely returned. "Most participants expect they'll find something useful to them, even though the researchers say not to anticipate anything actionable" (R-I 1). Participants may also overestimate the importance of IFs. "When [researchers] return things to people, it inevitably is with some imprimatur of authority that this may be important" (R-I 26).

\section{DISCUSSION}

We surveyed the genetics research community for their opinions on return of IFs and conducted in-depth interviews with selected researchers. Researchers overwhelmingly endorsed offering some types of IFs to research participants. Ninety-five percent of the survey respondents believed highly penetrant, clinically actionable results should be returned. This agrees with several consensus recommendations regarding return of IFs in genetic or genomic research. ${ }^{4,7,12}$ There was somewhat less agreement among researchers about whether IFs for conditions that were less penetrant, life-threatening, or clinically actionable should be offered to research participants-although a majority supported offering most kinds of IFs. Only 15\% of researchers endorsed returning a list of all variants to research participants, although this solution would allow participants direct access to their genetic data and limit the burden on the research community. However, researchers also expressed concerns about the burdens that returning IFs would place on the research enterprise, their lack of expertise in identifying the findings to be returned, and difficulties providing clinically relevant, accurate information across a broad range of conditions.

Researchers thought it would be helpful to develop a consensus list of clinically actionable, highly penetrant conditions, along with a well-curated, frequently updated database of established disease-causing mutations that would allow for robust and reliable interpretation of rare variants. ${ }^{13} \mathrm{~A}$ list of secondary findings that clinical diagnostic laboratories can use when reporting clinical WGS/WES results has been issued by the American College of Medical Genetics and Genomics; ${ }^{14}$ a modified list could be considered for the research community. Groups have also attempted to develop informatics approaches to automate analysis of IFs. ${ }^{15}$ Nonetheless, caution will be required in developing appropriate procedures because the number of reportable variants may be substantial ${ }^{16}$ and a surprisingly large number of apparently healthy people may have "pathogenic mutations" associated with uncommon syndromes (e.g., long QT syndrome). Either mutation databases are not robust, the penetrance for these mutations is much lower than previous estimates (especially in individuals without family histories), or both, leading to inaccurate conclusions released to participants. ${ }^{17}$ Well-curated, accurate-mutation databases for the genes for which IFs should be returned will be necessary before a general policy for return of IFs can be implemented.

Researchers' concerns may be mitigated somewhat by the reality that most studies are not systematically analyzing the genome to gather all clinically relevant variants. Therefore, they will probably only rarely identify IFs through their current analytical pipelines, reducing the burden of returning such data. The availability of local experts to provide education, genetic 
counseling, and medical care to participants to whom results might be returned will be an important part of reducing the remaining burden. The extent of burden actually entailed by returning IFs and the clinical benefit are deserving of study.

Respondents believed it would be important to make their policy on return of IFs clear during the consent process, and many were considering reobtaining consent from participants who were previously enrolled and are now being considered for WES/WGS. Policies for disclosing IFs in the consent process comport with recent recommendations about managing IFs in biobanks. ${ }^{18}$ We will be reporting elsewhere detailed findings regarding researchers' views on obtaining informed consent. Clearly, the development of educational materials, perhaps in online or video form, that would review the main topics for research participants to consider regarding return of IFs would remove some of the disclosure burden from investigators themselves.

Additional issues were relevant to our respondents when considering vulnerable populations such as children and fetuses. Researchers in general endorse returning fewer IFs concerning children and fetuses as compared with competent adults. However, it was striking that a large number of researchers considered returning IFs (presumably to parents) even for conditions that are not medically actionable or for which medical interventions should not be taken until adulthood. Recommendations from the American Academy of Pediatrics $^{19,20}$ and the American College of Medical Genetics and Genomics ${ }^{21,22}$ advise against predictive genetic testing for minors except when clinical actions can be taken during the pediatric period. These recommendations are aimed at preserving children's right to make decisions about receiving genetic information once they reach adulthood, and at preventing potential negative impacts of disclosure to parents on children's psychological development. However, the American College of Medical Genetics and Genomics recently issued recommendations to return medically actionable IFs to adults and children from clinical sequencing, including 57 conditions, among them adult-onset conditions without medical implications for children. ${ }^{14}$

\section{Conclusion}

Decisions regarding whether to return IFs and what findings to return necessarily reflect notions of research participants' moral rights to information about their genome, researchers' responsibilities to benefit their subjects and to avoid doing harm, and the effect of logistical constraints (e.g., lack of resources to confirm tests in Clinical Laboratory Improvement Amendments-approved labs or provide genetic counseling). Our data suggest widespread support for return of at least some IFs. However, there is uncertainty among investigators regarding which IFs should be offered and the process by which that should be accomplished. These results suggest that the research community would benefit from guidelines on return of IFs and the development of infrastructure to support that process.

\section{ACKNOWLEDGMENTS}

We thank the researchers for their contribution. This work was funded by National Human Genome Research Institute grants R01 HG006600, R21 HG006596, and P20 HG005535-01.

\section{DISCLOSURE}

The authors declare no conflict of interest.

\section{REFERENCES}

1. Kaufman D, Murphy J, Scott J, Hudson K. Subjects matter: a survey of public opinions about a large genetic cohort study. Genet Med 2008;10:831-839.

2. Wendler D, Emanuel $E$. The debate over research on stored biological samples: what do sources think? Arch Intern Med 2002;162:1457-1462.

3. Meacham MC, Starks H, Burke W, Edwards K. Researcher perspectives on disclosure of incidental findings in genetic research. J Empir Res Hum Res Ethics 2010;5:31-41.

4. Fabsitz RR, McGuire A, Sharp RR, et al. Ethical and practical guidelines for reporting genetic research results to study participants: updated guidelines from a National Heart, Lung, and Blood Institute working group. Circ Cardiovasc Genet 2010;3(6):574-580.

5. Affleck P. Is it ethical to deny genetic research participants individualised results? J Med Ethics 2009;35:209-213.

6. Cho MK. Understanding incidental findings in the context of genetics and genomics. J Law Med Ethics: J Am Soc Law Med Ethics. Summer 2008;36(2):280-285, 212

7. Commission NBA. Research Involving Human Biological Materials: Ethical Issues and Policy Guidance. National Bioethics Advisory Commission: Rockville, MD, 1999.

8. Van Ness B. Genomic research and incidental findings. J Law Med Ethics: J Am Soc Law Med Ethics. Summer 2008;36(2):292-297, 212.

9. McGuire AL, Caulfield T, Cho MK. Research ethics and the challenge of wholegenome sequencing. Nat Rev Genet 2008;9:152-156.

10. Corbin J, Strauss A. Basics of Qualitative Research: Techniques and Procedures for Developing Grounded Theory, 3rd edn. Sage Publications: Thousand Oaks, California, 2008.

11. Geertz C. Thick description: toward an interpretive theory of culture. In: The Interpretation of Cultures. Basic Books: New York, NY, 1973:3-30.

12. Beskow LM, Burke W, Merz JF, et al. Informed consent for population-based research involving genetics. JAMA 2001;286:2315-2321.

13. Evans JP, Rothschild BB. Return of results: not that complicated? Genet Med 2012;14:358-360.

14. Green RC, Berg JS, Grody WW, et al. ACMG recommendations for reporting of incidental findings in clinical exome and genome sequencing. American College of Medical Genetics and Genomics: Phoenix, Arizona, March 2013.

15. Berg JS, Adams M, Nassar N, et al. An informatics approach to analyzing the incidentalome. Genet Med 2013;15:36-44.

16. Johnston JJ, Rubinstein WS, Facio FM, et al. Secondary variants in individuals undergoing exome sequencing: screening of 572 individuals identifies highpenetrance mutations in cancer-susceptibility genes. Am J Hum Genet 2012;91:97-108.

17. Refsgaard L, Holst AG, Sadjadieh G, Haunsø S, Nielsen JB, Olesen MS. High prevalence of genetic variants previously associated with LQT syndrome in new exome data. Eur J Hum Genet 2012;20:905-908.

18. Wolf SM, Crock BN, Van Ness B, et al. Managing incidental findings and research results in genomic research involving biobanks and archived data sets. Genet Med 2012;14:361-384

19. Committee on Bioethics. Ethical issues with genetic testing in pediatrics. Pediatrics 2001;107(6):1451-1455.

20. Committee on Bioethics, Committee on Genetics, and The American College of Medical Genetics and Genomics Social, Ethical, Legal Issues Committee. Ethical and policy issues in genetic testing and screening of children. Pediatrics 2013;131:620-622.

21. Points to consider: ethical, legal, and psychosocial implications of genetic testing in children and adolescents. American Society of Human Genetics Board of Directors, American College of Medical Genetics Board of Directors. Am J Hum Genet 1995;57(5):1233-1241.

22. Ross LF, Saal HM, David KL, Anderson RR. Technical report: ethical and policy issues in genetic testing and screening of children. Genet Med 2013;15: $234-245$. 